\title{
Der Neuenburger Arzt Louis Guillaume (1833-1924), ein fast vergessener Pionier des staatlichen Gesundheitsdienstes*
}

\author{
Von Heinrich Buess
}

\begin{abstract}
Wenn man älter wird, muß man mit Bewußtsein auf einer gewissen Stufe stehen bleiben. Goethe, Maximen und Reflexionen (VII, 84)
\end{abstract}

Wer hätte vor fünfzig Jahren, als Adolf Faller und ich in der gleichen Klasse die Bänke der «Schule auf Burg» am Fuße des Basler Münsters drückten, daran gedacht, daß es uns beschieden sein würde, im gleichen Berufe und, erfüllt von gleichen Interessen, das Alter der Beschaulichkeit zu erreichen. Denn diese in der Goetheschen Maxime aufscheinende Überlegung ist es auch, die mich aus zwei Gründen veranlaßt hat, den an der Grenze zweier Sprachzonen aufgewachsenen Louis Guillaume als Thema für diesen Glückwunsch-Artikel zu wählen. Einmal im Hinblick auf den Jubilar, der sich ebenfalls zwischen deutscher und welscher Schweiz in überaus vielseitiger Weise als Arzt, Lehrer und Forscher theoretisch und praktisch betätigt hat, und dann aus der Sicht des Gratulanten, in dessen Garten die Pflanze herangewachsen ist, von der das hier präsentierte Sträußchen gepflückt wurde. Um das Bild zu erläutern und damit zugleich einem meiner väterlichen Freunde den Dank auch noch posthum abzustatten: Der Keim der Pflanze wurde wohl schon vor 40 Jahren gelegt, als Dr. med. Dr. h.c. Karl Reucker** (1890-1961), der Redaktor der noch heute unentbehrlichen «Ciba-Zeitschrift», eine Ausstellung über «Schweizer Ärzte als Forscher, Entdecker und Erfinder" plante und den Schreibenden damit beauftragte, in Form von hundert kurzen Bio-Ergographien die nötigen Unterlagen auch bibliographischer Natur beizusteuern. Diese «Bilder» entstanden in den Jahren 1943-44, und 60 Manuskripte wurden von Reucker ausgewählt und von seinen Mitarbeitern (vor allem Dr. phil. Walter Voegelin) als Texte für den Katalog der Ausstellung gestaltet. Die Bücher, Porträts und weitere Dokumente zeigte die Ciba von 1945 an in allen größeren Städten der Schweiz. Louis Guillaume kam damals nicht zu Ehren, sein Lebenswerk wurde aber in der mit Erwin H. Ackerknecht herausgegebenen «Kurzen Geschichte der großen Schweizer Ärzte» (Bern 1975) kurz zusammengefaßt. Hier folgt nun eine ausführliche Darstellung der wichtigsten Lebensstationen und Verdienste dieses heute vergessenen Mannes. Vielleicht verlockt unsere knappe Präsentation zu einer eingehenderen Beschäftigung mit Guillaume.

Louis Guillaume entstammte einem seit dem 16. Jahrhundert in Les Verrières (Val de Travers) urkundlich nachgewiesenen Neuenburger Geschlecht, das eine stattliche Zahl bedeutender Persönlichkeiten hervorgebracht hat. Mehrere unter diesen Männern haben sich politisch betätigt. Es scheint, daß die Söhne des Staatsrates Georges Guillaume (1817-1896) besonders begabt waren. So pflegte

\footnotetext{
* Prof. Dr. med. Adolf Faller (Fribourg) in alter Erinnerung zum 65. Geburtstag.

** Siehe dazu: Thomas Eduard Geigy, Dr. med. Dr. med.h.c. Karl Reucker, Diss. med. Zürich 1975.
} 
z. B. der revolutionär angehauchte Schriftsteller James (1844-1916) besondere Beziehungen zur Internationalen und zu Bakunin. Ein Neffe von Georges war der Physiker und Direktor des «Bureau international de poids et mesures» in Sèvres (1915), Charles-Edouard Guillaume. Er war korrespondierendes Mitglied des Institut de France und wurde 1920 mit dem Nobelpreis für Physik ausgezeichnet, denn er erfand die für die Zeitmeßtechnik wichtige Nickelstahllegierung Invar sowie weitere für die Uhrenindustrie grundlegende neue Metallmischungen und Neukonstruktionen.

Am 27. Februar 1923 sah die größte schweizerische Strafanstalt Witzwil (Kanton Bern) eine eigenartige Gesellschaft in ihren Mauern versammelt. Hochgeehrte Gäste aus den Kreisen des Strafvollzuges und Strafrechts sowie ein Abgeordneter der Neuenburger Regierung waren zusammengekommen. Es galt, eine der bedeutendsten Gestalten aus dem einstigen öffentlichen Leben der Eidgenossenschaft gebührend zu ehren. Vertraute Freunde wollten einem 90 jährigen den Dank ungezählter Mitmenschen abstatten, in deren Leben das freundliche Wesen des Jubilars wie ein erhellender und wärmender Sonnenstrahl hineingedrungen war. Dieser Mittelpunkt der in aller Stille vereinten Schar von Männern war niemand anderes als Louis Guillaume, der tatkräftige Pionier des neuenburgischen Gesundheitswesens, dem Ständerat Ernest Béguin die Grüße der Regierung überbrachte, der unentwegte Freund der Strafgefangenen, den der Gastgeber, Otto Kellerhals, hochleben ließ, der ideenreiche Förderer der Demographie in der Schweiz, dessen Verdienste Marcel Mey, der Vorsteher des eidgenössischen statistischen Amtes, in beredten Worten würdigte. Eine Anregung, es solle dem Bild des Gefeierten in jeder Strafanstalt ein Ehrenplatz eingeräumt werden, fand vollen Beifall und wurde mancherorts in die Tat umgesetzt.

\section{I}

Ein reiches Leben lag hinter dem unscheinbar gewordenen Greis, dessen warme, immer noch helle Augen das einstige Feuer verrieten. Hart an der französischen Grenze, dort, wo nicht ganz 40 Jahre später die traurigen Überreste der Armee Bourbaki von den Soldaten General Herzogs in Empfang genommen werden sollten, in Les Verrières, kam Guillaume am 27. Februar 1833 als Sohn eines Notars zur Welt. Die abwechslungsreiche Juralandschaft des einzigartigen Val de Travers muß auf den Knaben einen tiefen Eindruck gemacht haben. Sein Lebtag kam er nicht davon los. Denn kaum der Dorfschule entwachsen, mußte Guillaume in die Welt hinaus. In Basel, weit entfernt von zu Hause, besuchte er das Gymnasium. Den Weg dorthin soll er nach den Ferien mehrmals zu Fuß zurückgelegt haben. So durchwanderte er schon in jungen Jahren den vielgestaltigen 
Neuenburger und Berner Jura. Das Studium der Medizin absolvierte er in Zürich, wo er, noch nicht 22 jährig, mit einer Dissertation über die Zuckerkrankheit den Doktorgrad erwarb (1854).

Nachdem sich der allzu junge Arzt noch etwas in der Welt umgesehen hatte Wien, Berlin, Paris und London waren die wichtigsten Stationen seiner Studienreise - eröffnete er eine medizinische Praxis in Neuenburg, und zwar im Schicksalsjahr 1856, das dem Leben dieser Stadt als preußischem Fürstentum ein Ende machte. Es wäre nicht verwunderlich, wenn Guillaume, der später Mitglied der radikalen Partei war, damals auf der Seite der Aufständischen mitgekämpft hätte. Jedenfalls muß er ein rechter Draufgänger gewesen sein, wie sein öffentliches Auftreten zeigt, das nicht lange auf sich warten ließ. Die Wahl zum Mitglied des Großen Rates jedoch nahm er wohl nur an, um seinen Plänen zur Verwirklichung zu helfen.

Vorerst waren es Guillaumes Freude an den Schönheiten seines geliebten Jura und das Interesse an der so wechselvollen Geschichte seiner Heimat, die ihn die ersten Schritte in die Öffentlichkeit tun ließen. Guillaume war nicht der kühne Denker, der aus der Fülle seines Geistes neue Ideen hervorbringen konnte. Dagegen besaß er die Gabe, durchaus selbständig Anregungen von außen auf seine besonderen Verhältnisse zu übertragen. Am 19. April 1863 wurde in Olten der Schweizerische Alpenclub gegründet mit dem Ziel, die Alpen namentlich in geographischer, naturwissenschaftlicher und landschaftlich-malerischer Richtung genauer kennenzulernen. Was lag da für den von seinem Jura begeisterten Guillaume näher, als eine ähnliche Vereinigung in Neuenburg ins Leben zu rufen. Vor allem aber wollte der von erzieherischen Idealen erfüllte Arzt die Schuljugend mit den Schönheiten und den historischen Stätten der Heimat vertraut machen. Nachdem er schon seit längerer Zeit mit einigen Schülern zweimal wöchentlich zusammengekommen war, um aufs Land hinaus zu wandern, erhielt das ganze Vorhaben festere Gestalt durch die Beiziehung der Lehrerschaft.

Am 21. Mai 1865 fand die eigentliche Gründungsversammlung des «Club Jurassien », wie er sich nannte, in einem Wäldchen nahe bei Neuenburg statt. Durch Pflanzen- und Tiersammlungen, Herstellung von Unterrichtstafeln, durch Abfassen kleiner Aufsätze über selbständige Beobachtungen sollte unter den reiferen Schulkindern ein engerer Kontakt mit den Schönheiten der Natur hergestellt werden. Doch erweiterte sich später der Aufgabenkreis nach der wissenschaftlichen Seite hin. Dies beweist auch die schon bald erfolgte Gründung einer eigenen Zeitschrift; es war der durch Originalität hervorstechende, die lokale Naturforschung nachhaltig befruchtende «Rameau de Sapin» (Tannenzweig). Mit Guil- 


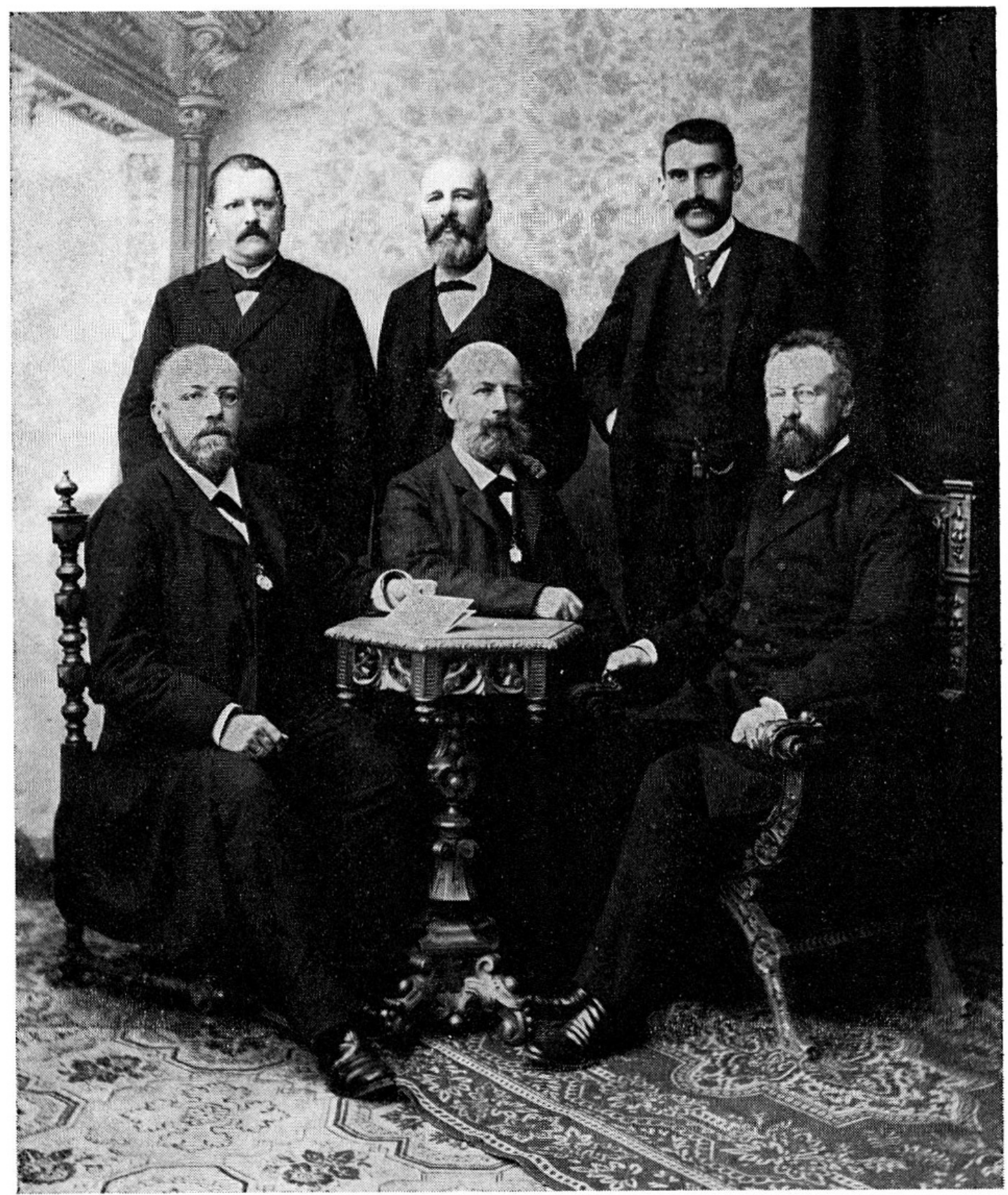

Schweizer Teilnehmer am Gefängniskongreß in Petersburg 1890.

Hinten: Franz La Roche (1847-1912), Gustave Correvon (1841-1911) und Ferdinand Rüsch (1863-1937). Vorne: Bernhard Riggenbach (1848-1895), Louis Guillaume (1833-1924) und Karl Stooss (1849-1934). Univ.-Bibl. Basel, Sammelporträt 1272 
laume zusammen gehörte auch Professor Louis Favre (1822-1904), der Neuenburger Pädagoge, Zeichner, Naturforscher, Biograph, Novellist und Historiker, zu den Freunden des Clubs. Nach dem Vorbild des Genfers Rodolphe Töpffer (1799-1846) ließ Guillaume die Erlebnisse der belehrenden und unterhaltenden Fußtouren in feinsinnigen Alben durch Künstlerhand festhalten; so wurde z.B. eine Wanderung auf die Schlachtfelder von Grandson und Murten, geführt von Oberst Louis-Alphonse de Mandrot, vom bekannten Maler Auguste Bachelin in eindrucksvollen Zeichnungen verewigt. Bedeutende Naturforscher, Historiker, Künstler und Offiziere liehen dem jungen Werk ihre Unterstützung. Dieses hat zur Verbreitung der naturwissenschaftlichen Kenntnisse in großen Teilen der welschen Schweiz nicht wenig beigetragen.

Hatte Guillaume schon bei diesem Unternehmen sein geschichtliches Interesse in hohem Maße geleitet, so war dies ganz ausgesprochen der Fall bei der Gründung der neuenburgischen Gesellschaft für Geschichte und Archäologie am 3. April 1864, an der Guillaume ebenfalls hervorragend beteiligt ist. Auf die Anregung des Buchdruckers Marolf, eine Sammlung geschichtlicher Arbeiten anzulegen, taten sich damals einige Männer zusammen, darunter außer Favre, Bachelin und dem um die Medizingeschichte Neuenburgs verdienten Spitalarzt Edouard Cornaz (1825-1904) auch der überragende Forscher Edouard Desor (1811-1882). Guillaume gehörte seit 1865 der Redaktionskommission des neugegründeten «Musée Neuchâtelois » an, das sich zu einer angesehenen Zeitschrift entwickelt hat. und amtierte 1870 als Präsident der geschichtsforschenden Gesellschaft. Während etwa eines Vierteljahrhunderts erschienen aus Guillaumes Feder gegen 40 Mitteilungen, die alle möglichen Gebiete berührten, von den ersten Dampfschiffen bis zu der Einführung des Kaffees in Neuenburg.

Diese beiden Schöpfungen mögen Guillaumes außerordentliche Vielseitigkeit zeigen, die ihm durch sein ganzes Leben erhalten geblieben ist. Indessen war er doch in erster Linie Arzt, und auf medizinischem Gebiet liegen seine großen Verdienste. Bald erhielt er bei seiner praktischen Tätigkeit tiefe Einblicke in die Unterlassungen auf hygienischem Gebiet, vor allem unter den Schulkindern. So machte er sich voll Tatkraft daran, die Mißstände an der Wurzel anzupacken. Auch um die verwahrloste Jugend nahm er sich an und beschäftigte sich schon früh mit den Fragen des Gefängniswesens, der Fürsorge für die aus der Haft entlassenen Sträflinge, wie ihm überhaupt das Schicksal der von der Öffentlichkeit wenig beachteten kleinen Leute ein brennendes Problem bedeutete. Seit den ersten Jahren der praktischen Wirksamkeit befaßte sich Guillaume auch mit der Demographie, d.h. der beschreibenden Richtung der Bevölkerungsstatistik, einem Gebiet, das zur Hygiene enge Beziehungen aufweist. Alle diese anfänglich 
Aussicht stellten. Unter den damaligen Umständen nahm Guillaume den Ruf in den Dienst des Bundes an (1889). War er schon früher als Delegierter des Bundesrates zu internationalen Kongressen des Gefängniswesens abgeordnet worden, so nahm jetzt seine Tätigkeit auf internationalem Boden immer mehr zu: 1893 wurde er zum ständigen Sekretär der internationalen Gefängniskommission ernannt und hatte in dieser Stellung die Vorbereitung und Berichterstattung der Kongresse zu besorgen, eine Tätigkeit, die seinen Namen in den Fachkreisen aller vertretenen Länder bekannt machte. Auch an Hygienekongressen und an den Tagungen der statistischen Gesellschaften nahm er in höchster Stellung teil. Seine Voten galten viel unter den Prominenten der verschiedenen Staaten. Unter seiner Leitung nahm das statistische Amt einen gewaltigen Aufschwung. Bei Guillaumes Amtsantritt waren 30 Leute beschäftigt; 1912 waren es mehr als 100 Angestellte. Auch in dieser Stellung spürten nicht nur die Untergebenen, sondern alle, die mit Guillaume zu tun hatten, wie sehr Güte und Zuvorkommenheit dessen Wesen beherrschten und ihn alle seine Obliegenheiten aufs gewissenhafteste erfüllen ließen.

Im Jahre 1913 trat Guillaume von seiner Stellung zurück. 80 Jahre alt war er geworden im Dienste für das Wohl seiner Mitbürger. Bei seinem Sohn, dem Kunstmaler Louis Guillaume, in Epagnier, brachte er in aller Zurückgezogenheit die letzten Jahre seines Lebens zu. Dort, an den Ufern der Thièle, angesichts der alten Fundstätte «La Tène», durfte er sich noch lange des dankbaren Gedenkens seiner Freunde und Fachgenossen erfreuen und wissen, daß er manchem Mitmenschen den richtigen Weg ins Leben gewiesen hatte. Einige Wochen, nachdem er einen Hirnschlag erlitten hatte, starb Guillaume am 25. Januar 1924. in Epagnier im patriarchalischen Alter von fast 91 Jahren.

«Bei vielen, die Guillaume gekannt haben, wird das Erinnerungsbild an sein Wissen und Können im Laufe der Zeiten verblassen; eines aber wird in jedem Gedächtnis dauernd haften: die Erinnerung an seine Milde, seine liebenswürdige Gesinnung» (E. W. Milliet). «Ich habe nur ganz wenige Männer gesehen, in deren Antlitz mir Güte und Gescheitheit so schön gepaart erschienen » (Gustav Schmoller). So äußerten sich führende Vertreter der Wissenschaft über Louis Guillaume.

\section{II}

Die Verdienste Guillaumes reihen sich in der chronologischen Reihenfolge am natürlichsten aneinander. Seinem Aufstieg von Stufe zu Stufe in der schweizerischen Hierarchie des öffentlichen Lebens folgend, erkennen wir, wie sich sein Wirken in immer fruchtbarerer Weise entfalten konnte.

Als Student war Guillaume mit den gewaltigen Errungenschaften bekannt geworden, die auf hygienischem Gebiet in England erreicht worden waren. Wie weit 
stand seine Heimat noch dahinter zurück! Eine einheitliche Gesetzgebung, wie sie um die Mitte des 19. Jahrhunderts Großbritannien erhalten hatte, konnte allein zum Ziele führen. Nach seiner Rückkehr in die Heimat verlor Guillaume dieses hohe Ziel nicht mehr aus den Augen. Als Mitglied der Sanitätskommission beeinflußte er in maßgebender Weise die Abfassung der Gesetze über die Gesundheitspolizei, die zu den Kompetenzen der kantonalen Regierung gehört. 1862 wurde in einem ersten Gesetz das Vergehen gegen die öffentliche Gesundheit unter Strafe gestellt; 1864 und 1868 wurden in den Gemeinden die nötigen Dienststellen für das Sanitätswesen geschaffen, die auf Grund der Erfahrungen in Zürich (Choleraepidemie von 1867) zweckentsprechend organisiert und eingeübt wurden. Eine neue Gelegenheit bot sich Guillaume, als er 1878 an den auf seinen Antrag geschaffenen Lehrstuhl als Professor der Hygiene berufen wurde. In den Hörsälen der Neuenburger Akademie vereinigten sich neben den Ärzten und Apothekern auch die Pfarrer und Lehrer als maßgebende Vertreter der Gemeinden, um Guillaumes Anweisungen in den verschiedenen Zweigen der Hygiene entgegenzunehmen. Für die freiwillige Weiterbildung schuf er die heute noch bestehenden «Feuilles d'hygiène». So weckte er das allgemeine Interesse und die freudige Mitarbeit an den wichtigen Maßnahmen zur Verhütung der Krankheiten. Und der Kanton Neuenburg erhielt als erster eine umfassende Organisation der öffentlichen Gesundheitspflege. Guillaumes Überblick von 1881 vermittelt ein gutes Bild von dem, was in den Jahren seines öffentlichen Wirkens erreicht worden ist.

Einer der Zweige der Volkswohlfahrt, die Schulhygiene, erfuhr besondere Förderung durch Guillaume, bei dem die Interessen der Jugend allen übrigen vorangingen. Seine Erfahrungen und Vorschläge für die Besserung der betrüblichen Zustände legte er in verschiedenen Schriften nieder. Die bedeutendste unter ihnen, die 1864 erschienene «Hygiène scolaire», fand ein starkes Echo nicht nur unter der Neuenburger Bevölkerung, sondern bis weit über die Kantons-, ja die Landesgrenzen hinaus. Sie lenkte die Aufmerksamkeit auf ein Gebiet, das bisher allzusehr vernachlässigt worden war, und wurde zum Ausgangspunkt einer tiefgreifenden Reform. Noch in dem ein Vierteljahrhundert später erschienenen 6. Band des Handbuches der Hygiene von Theodor Weyl (1851-1913) wurde das Werk von Guillaume als klassisch gepriesen. In einer späteren Schrift verwendet sich der Neuenburger Arzt für eine Verbesserung der Schulhäuser in baulicher Hinsicht (1874).

Die Gefängnisstrafe kam im Rechtswesen der Schweiz vereinzelt schon im späteren Mittelalter vor. Als Pioniere der Gefängnisreform im Zeitalter der Aufklärung dürfen der englische Philanthrop John Howard (1726-1790) und der französische Naturforscher Antoine-Laurent Lavoisier (1743-1794) gelten. Be- 
deutende Fortschritte im Gefängniswesen brachte aber erst das 19. Jahrhundert, obgleich die von Napoleon angestrebte Strafrechtseinheit nach der Mediation rasch wieder verlorenging. In Lausanne und Genf entstanden die ersten modernen Strafanstalten auf Schweizerboden. Guillaume bezog von Anfang an das Gebiet des Strafvollzuges in seine Reformbestrebungen ein. Auch hier galten der Jugend die ersten Sorgen. Nachdem er 1872 auf der Jahresversammlung des Schweizerischen Vereins für Strafwesen in Neuenburg einen geschichtlichen Abriß über das kantonale Gefängniswesen gegeben hatte, beantragte Guillaume drei Jahre später bei der Schweizerischen Gemeinnützigen Gesellschaft die Gründung einer interkantonalen Rettungsanstalt für die verwahrloste und kriminelle Jugend und sprach sich 1906 für die Schaffung von Kindergerichtshöfen aus, wie sie in Nordamerika schon bestanden. Diese Forderungen klangen in jener Zeit wie Heroldsrufe der dann einsetzenden Bewegung zur Umgestaltung des Jugendstrafrechts in ein Jugendfürsorgerecht. Und heute sind Guillaumes Postulate auf Schweizerboden weitgehend verwirklicht.

1875 unterbreitete Guillaume der Berner Regierung ein Gutachten über die «Reorganisation des Straf- und Gefängniswesens im Kanton Bern». In seiner Funktion als Strafanstaltsdirektor war Guillaume bemüht um die noch heute aktuellen Fragen des Arbeitsverdienstanteils der Gefangenen und um die Weiterbildung des Personals. Am eindrücklichsten bewies Guillaume seine Fähigkeiten als Sekretär der internationalen Kommission für das Gefängniswesen. Wenn man bedenkt, daß die Vorbereitung der Kongresse zu seinen Pflichten gehörte und daß die zu erstattenden Berichte über einen einzigen Kongreß oft mehrere dicke Bände füllten, so kann man sich einen Begriff machen von Guillaumes hingebungsvoller Tätigkeit. In den Jahren 1873-1910 war er Delegierter an acht Kongressen verschiedener Art, und 1893-1913 bildete er als Sekretär die eigentliche Seele der Kongresse, deren Berichterstattung für das schweizerische Strafwesen zur Quelle erfreulichster Belebung in Wissenschaft und Praxis wurde.

Der statistische Gedanke im Gebiet der heutigen Schweiz geht auf das Altertum zurück, wenigstens in der Form der Volkszählung (Zählung der Helvetier zur Zeit Cäsars). Im 17. Jahrhundert sind dann die Ergebnisse einzelner Volkszählungen genauer bekannt geworden, die allerdings immer nur einzelne Kantone umfaßten (darunter taten sich namentlich Zürich, Basel und Bern hervor). Im 19. Jahrhundert, das auch die erste schweizerische Volkszählung brachte, wurde von einigen weitblickenden Männern die Statistik erheblich gefördert. Unter den Gelehrten stehen obenan der Basler Professor Christoph Bernoulli (1782-1863) mit seinem «Archiv für Statistik und Nationalökonomie», dann der Zürcher Jakob Heinrich Hottinger (1815-1876) und Jean Picot (1777-1864) mit seiner 
großen «Statistique de la Suisse» (1819). Mehr als diese Theoretiker, zu denen dann besonders der Genfer Jacob-Marc d'Espine (1805-1860) zu zählen ist, trugen zur Förderung der neuen Wissenschaft die Politiker bei, die ihre Pläne in die Tat umzusetzen verstanden. Hier ist vor allem der Name des ersten Tessiner Bundesrates Stefano Franscini (1796-1857) zu nennen, der am Gelingen der ersten Volkszählung großen Anteil hatte. Seine 1827 erschienene «Statistica della Svizzera » sollte von großer Bedeutung für den Ausbau der amtlichen Statistik werden. Auf Franscini geht ohne Zweifel auch die Schaffung eines eidgenössischen statistischen Bureaus zurück, das 1860 von den Räten beschlossen wurde. In demselben Jahr wurde die periodische Volkszählung (alle 10 Jahre im Dezember) gesetzlich verankert. Im früheren Berner Regierungsrat Johann Jakob Kummer (1828-1913) fand sich endlich auch der geeignete Mann für die durchgreifende Reorganisation des neuen Bundesamtes, dessen Tätigkeitsgebiet durch die Verfassung von 1874 erheblich erweitert wurde. Nach Kummers Übertritt ins Versicherungsamt (1885) übernahm der Basler Edmund Wilhelm Milliet (1857-1931) für kurze Zeit die Leitung des statistischen Amtes.

Guillaume brachte als Arzt von 1890 an einen neuen Geist in die tote Materie. Getreu seinem Votum auf dem Hygiene-Kongreß in Budapest: «Auf allen Gebieten der Demographie kommt der Krankheit erhebliche Bedeutung zu, sie darf als Faktor von grundlegender Wichtigkeit niemals vernachlässigt werden », richtete er sein Augenmerk vor allem auf den Ausbau der medizinischen Seite der Statistik. Aber auch anthropologische Merkmale gehörten in Guillaumes Interessenkreis, wie seine Untersuchung über die Farbe der Augen und der Haare der Schulkinder (1876) beweist, übrigens eine der frühesten Sammelstatistiken auf diesem jungen Gebiet. Guillaume ist denn auch die eigentliche Schöpfung der Todesursachen-Statistik zu verdanken, die so wertvoll ist für den Fortschritt der medizinischen Wissenschaft. Von 1891 an erschien auf seine Initiative hin das aufschlußreiche und heute unentbehrlich gewordene «Statistische Jahrbuch der Schweiz». Unter Guillaumes Leitung wurden die ersten Zählungen der Künste und Gewerbe durchgeführt. Besonders lag ihm selbstverständlich das Gefängniswesen am Herzen; er rief die nach Kantonen vorgenommene statistische Erfassung der Gefängnisse und ihrer Insassen ins Leben (1892-1896), deren Ergebnisse er im Jahre 1900 veröffentlichte. Ähnliche Zählungen veranlaßte er auch 1906. Bedauerlicherweise gingen Guillaumes Absichten nicht in Erfüllung, die monatlichen Bulletins über den Stand der Gefängnisbevölkerung (seit 1890) definitiv einzuführen; sie wurden 1920 gänzlich aufgehoben. Der Wissenschaftler Guillaume ging wohl in seinen Forderungen so weit, daß ihre praktische Durchführung nicht mehr Schritt halten konnte. Immerhin bildeten seine Vorarbeiten 
die zahlenmäßige Grundlage für die Schaffung einer Strafrechtseinheit in der Schweiz. Daher war Guillaumes Rat als Mitglied der ersten Expertenkommission für das schweizerische Strafgesetzbuch sehr geschätzt. Auch die Erfassung der Schwachsinnigen und Krüppel im schulpflichtigen Alter war von größtem Wert. Schließlich sei noch an die ehrenamtliche Arbeit Guillaumes als Redaktor der «Zeitschrift für Schweizerische Statistik und Volkswirtschaft» erinnert, die er von 1890 bis zu seinem Rücktritt geleistet hat.

Guillaumes Leistungen seien zusammengefaßt in den schönen Worten, die ihm der hervorragende Strafrechtslehrer Emil Zürcher zum 90. Geburtstag zurief: «Sie können mit Stolz und Befriedigung auf ein an Arbeit überreiches Leben zurückblicken. Vieles, was Sie erstrebt, hat sich herrlich erfüllt, für anderes stehen wir noch mitten im Kampfe.»

\section{Bibliographie (Auswahl)}

1. Beiträge zur Lehre der Zuckerausscheidung im Diabetes mellitus, Diss. med. Zürich 1854 (37 S.).

2. Borel, Eugène, und Guillaume, Louis, Frédéric Rössinger, Neuchâtel 1863.

3. Hygiène scolaire. Considérations sur l'état hygiénique des écoles publiques présentées aux autorités scolaires, aux institutions et aux parents. Genève et Paris 1864 (128 S.). (2.Aufl. 1865; deutsch: Aarau 1865.)

4. Rapport au conseil d'état de la république et canton de Neuchâtel sur les mesures sanitaires prises à Zurich pendant l'épidémie du choléra, La Chaux-de-Fonds 1868 (84. S.).

5. Hygiène des écoles; conditions architecturales et économiques, Paris 1874 (66 S.).

6. Statistique des corps de Gendarmerie en Suisse accompagnée de quelques réflexions sur la police et sur les moyens d'améliorer ce service publique, Bern 1874.

7. Über die Errichtung einer interkantonalen Rettungsanstalt für junge Verbrecher und verwahrloste Kinder, die das 13. Altersjahr zurückgelegt haben, Bern 1875 (Bericht an den Schweiz. Verein für Straf- und Gefängniswesen).

8. Die Reorganisation des Straf- und Gefängniswesens des Kanton Bern. Gutachten. Bern 1875.

9. Observations sur la couleur des yeux et des cheveux dans le canton de Neuchâtel. In: Bull. de la Soc. des Sc. nat. de Neuch. 1876.

10. Coup d'œil sur la vie sociale dans le canton de Neuchâtel; liste des institutions et des sociêtés libres de bienfaisance, d'utilité, d'éducation, d'instruction et de récréation, Neuchâtel 1881 (215 S.).

11. Le vaccin Jennérien et le vaccin animal; rapport présenté au nom de la commission de santé à la direction de l'intérieur, Neuchâtel 1881 (51 S.).

12. L'épidémie de variole dans le canton de Neuchâtel en 1880, Neuchâtel 1881 (75 S.).

13. L'eau du Seyon et la fièvre typhoïde à Neuchâtel, Neuchâtel 1882.

14. L'hygiène. Landesausstellung 1883. Bericht über Gruppe 31. Zürich 1884.

15. L'enseignement scolaire dans les pénitenciers de la Suisse, Bern 1886. 


\section{Biographie}

1. D[avid] Widmer, Verh. des Schweiz. Vereins für Straf-, Gefängniswesen und Schutzaufsicht, N. F., H. 4 (1924), S. 53-61 (Portr., bibliogr. Angaben).

2. E[mil] Zürcher, Schweiz. Ztschr.f. Strafrecht 36 (1923), S.1-5.

3. Le Rameau de Sapin 1924, S. 25-36 (Eine Sammlung von Zeitungsnekrologen, gutes Porträt).

4. A[rmand] du Pasquier, Musée Neuchâtelois, N. S.11 (1924), S.47-48 (Portr.).

5. Zeitschrift für Schweizerische Statistik und Volkswirtschaft 1923, S. 3 f. (F[ritz] Mangold); 1924, S. 89 (E[dmund] W[ilhelm] Milliet).

6. Messager Boiteux de Neuchâtel pour l'an 1925, S.43-44 (Portr.).

\section{Summary}

This short biographical essay is an attempt to delineate the various activities in public life of the physician Louis Guillaume (1833-1924), a descendent of a distinguished family in the canton Neuchâtel. His most outstanding merits which were also internationally appreciated are in the fields of public hygiene, water purification, school health service, prison reform, penal institutions and demographic statistics. He finally was director of the statistical office of Switzerland.

Prof. Dr. med. Heinrich Buess

Medizinhistorische Bibliothek

Schönbeinstraße 40

4056 Basel 